\title{
The sum of degrees in cliques
}

\author{
Béla Bollobás* ${ }^{* \ddagger}$ and Vladimir Nikiforov* \\ Submitted: Sep 10, 2004; Accepted: Nov 1, 2005; Published: Nov 7, 2005 \\ Mathematics Subject Classifications: 05C35
}

\begin{abstract}
For every graph $G$, let

$$
\Delta_{r}(G)=\max \left\{\sum_{u \in R} d(u): R \text { is an } r \text {-clique of } G\right\}
$$
\end{abstract}

and let $\Delta_{r}(n, m)$ be the minimum of $\Delta_{r}(G)$ taken over all graphs of order $n$ and size $m$. Write $t_{r}(n)$ for the size of the $r$-chromatic Turán graph of order $n$.

Improving earlier results of Edwards and Faudree, we show that for every $r \geq 2$, if $m \geq t_{r}(n)$, then

$$
\Delta_{r}(n, m) \geq \frac{2 r m}{n},
$$

as conjectured by Bollobás and Erdős.

It is known that inequality (1) fails for $m<t_{r}(n)$. However, we show that for every $\varepsilon>0$, there is $\delta>0$ such that if $m>t_{r}(n)-\delta n^{2}$ then

$$
\Delta_{r}(n, m) \geq(1-\varepsilon) \frac{2 r m}{n}
$$

\section{Introduction}

Our notation and terminology are standard (see, e.g. [1]): thus $G(n, m)$ stands for a graph of $n$ vertices and $m$ edges. For a graph $G$ and a vertex $u \in V(G)$, we write $\Gamma(u)$ for the set of vertices adjacent to $u$ and set $d_{G}(u)=|\Gamma(u)|$; we write $d(u)$ instead of $d_{G}(u)$ if the graph $G$ is understood. However, somewhat unusually, for $U \subset V(G)$, we set $\widehat{\Gamma}(U)=\left|\cap_{v \in U} \Gamma(v)\right|$ and $\widehat{d}(U)=|\widehat{\Gamma}(U)|$.

We write $T_{r}(n)$ for the $r$-chromatic Turán graph on $n$ vertices and $t_{r}(n)$ for the number of its edges.

\footnotetext{
* Department of Mathematical Sciences, University of Memphis, Memphis TN 38152, USA

†Trinity College, Cambridge CB2 1TQ, UK

${ }^{\ddagger}$ Research supported in part by DARPA grant F33615-01-C-1900.
} 
For every $r \geq 2$ and every graph $G$, let $\Delta_{r}(G)$ be the maximum of the sum of degrees of the vertices of an $r$-clique, as in the abstract. If $G$ has no $r$-cliques, we set $\Delta_{r}(G)=0$. Furthermore, let

$$
\Delta_{r}(n, m)=\min _{G=G(n, m)} \Delta_{r}(G) .
$$

Since $T_{r}(n)$ is a $K_{r+1}$-free graph, it follows that $\Delta_{r}(n, m)=0$ for $m \leq t_{r-1}(n)$. In 1975 Bollobás and Erdős [2] conjectured that for every $r \geq 2$, if $m \geq t_{r}(n)$, then

$$
\Delta_{r}(n, m) \geq \frac{2 r m}{n} .
$$

Edwards [3], [4] proved (2) under the weaker condition $m>(r-1) n^{2} / 2 r$; he also proved that the conjecture holds for $2 \leq r \leq 8$ and $n \geq r^{2}$. Later Faudree [7] proved the conjecture for any $r \geq 2$ and $n>r^{2}(r-1) / 4$.

For $t_{r-1}(n)<m<t_{r}(n)$ the value of $\Delta_{r}(n, m)$ is essentially unknown even for $r=3$ (see [5], [6] and [7] for partial results.) A construction due to Erdős and Faudree (see [7], Theorem 2) shows that, for every $\varepsilon>0$, there exists $\delta>0$ such that if $t_{r-1}(n)<m<$ $t_{r}(n)-\delta n^{2}$ then

$$
\Delta_{r}(n, m) \leq(1-\varepsilon) \frac{2 r m}{n} .
$$

The construction is determined by two appropriately chosen parameters $a$ and $d$ and represents a complete $(r-1)$-partite graph with $(r-2)$ chromatic classes of size $a$ and a $d$-regular bipartite graph inserted in the last chromatic class.

In this note we prove a stronger form of (2) for every $r$ and $n$. Furthermore, we prove that $\Delta_{r}(n, m)$ is "stable" as $m$ approaches $t_{r}(n)$. More precisely, for every $\varepsilon>0$, there is $\delta>0$ such that if $m>t_{r}(n)-\delta n^{2}$ then

$$
\Delta_{r}(n, m) \geq(1-\varepsilon) \frac{2 r m}{n}
$$

for $n$ sufficiently large.

\subsection{Preliminary observations}

If $M_{1}, \ldots, M_{k}$ are subsets of a (finite) set $V$ then

$$
\left|\cap_{i=1}^{k} M_{i}\right| \geq \sum_{i=1}^{k}\left|M_{i}\right|-(k-1)|V| .
$$

The size $t_{r}(n)$ of the Turán graph $T_{r}(n)$ is given by

$$
t_{r}(n)=\frac{r-1}{2 r} n^{2}-\frac{s}{2}\left(1-\frac{s}{r}\right) .
$$

where $s$ is the remainder of $n$ modulo $r$. Hence,

$$
\frac{r-1}{2 r} n^{2}-\frac{r}{8} \leq t_{r}(n) \leq \frac{r-1}{2 r} n^{2} .
$$




\section{A greedy algorithm}

In what follows we shall identify a clique with its vertex set.

Faudree [7] introduced the following algorithm $\mathcal{P}$ to construct a clique $\left\{v_{1}, \ldots, v_{k}\right\}$ in a graph $G$ :

Step 1: $v_{1}$ is a vertex of maximum degree in $G$;

Step 2: having selected $v_{1}, \ldots, v_{i-1}$, if $\widehat{\Gamma}\left(v_{1}, \ldots, v_{i-1}\right)=\varnothing$ then set $k=i-1$ and stop $\mathcal{P}$, otherwise $\mathcal{P}$ selects a vertex of maximum degree $v_{i} \in \widehat{\Gamma}\left(v_{1}, \ldots, v_{i-1}\right)$ and step 2 is repeated again.

Faudree's main reason to introduce this algorithm was to prove Conjecture (2) for $n$ sufficiently large, so he did not study $\mathcal{P}$ in great detail. In this section we shall establish some properties of $\mathcal{P}$ for their own sake. Later, in Section 3, we shall apply these results to prove an extension of (2) for every $n$.

Note that $\mathcal{P}$ need not construct a unique sequence. Sequences that can be constructed by $\mathcal{P}$ are called $\mathcal{P}$-sequences; the definition of $\mathcal{P}$ implies that $\widehat{\Gamma}\left(v_{1} \ldots v_{k}\right)=\varnothing$ for every $\mathcal{P}$-sequence $v_{1}, \ldots, v_{k}$.

Theorem 1 Let $r \geq 2, n \geq r$ and $m \geq t_{r}(n)$. Then every graph $G=G(n, m)$ is such that:

(i) every $\mathcal{P}$-sequence has at least $r$ terms;

(ii) for every $\mathcal{P}$-sequence $v_{1}, \ldots, v_{r}, \ldots$,

$$
\sum_{i=1}^{r} d\left(v_{i}\right) \geq(r-1) n
$$

(iii) if equality holds in (5) for some $\mathcal{P}$-sequence $v_{1}, \ldots, v_{r}, \ldots$ then $m=t_{r}(n)$.

Proof Without loss of generality we may assume that $\mathcal{P}$ constructs exactly the vertices $1, \ldots, k$ and hence $d(1) \geq \ldots \geq d(k)$.

Proof of (i) and (ii) To prove (i) we have to show that $k \geq r$. For every $i=1, \ldots, k$, let $M_{i}=\Gamma(i)$; clearly,

$$
\sum_{i=1}^{k} d(i) \leq(q-1) n,
$$

since, otherwise, (3) implies that $\widehat{\Gamma}\left(v_{1} \ldots v_{k}\right) \neq \varnothing$, and so $1, \ldots, k$ is not a $\mathcal{P}$-sequence, contradicting the choice of $k$. Suppose $k<r$, and let $q$ be the smallest integer such that the inequality

$$
\sum_{i=1}^{h} d(i)>(h-1) n
$$

holds for $h=1, \ldots, q-1$, while

$$
\sum_{i=1}^{q} d(i) \leq(q-1) n .
$$


Clearly, $1<q \leq k$.

Partition $V=\cup_{i=1}^{q} V_{i}$, so that

$$
\begin{aligned}
V_{1} & =V \backslash \Gamma(1), \\
V_{i} & =\widehat{\Gamma}([i-1]) \backslash \widehat{\Gamma}([i]) \quad \text { for } i=2, \ldots, q-1, \\
V_{q} & =\widehat{\Gamma}([q-1]) .
\end{aligned}
$$

We have

$$
\begin{aligned}
2 m & =\sum_{j \in V} d(j)=\sum_{h=1}^{q} \sum_{j \in V_{h}} d(j) \leq \sum_{i=1}^{q} d(i)\left|V_{i}\right| \\
& =d(1)(n-d(1))+\sum_{i=2}^{q-1} d(i)(\widehat{d}([i-1])-\widehat{d}([i]))+d(q) \widehat{d}([q-1]) \\
& =d(1) n+\sum_{i=1}^{q-1} \widehat{d}([i])(d(i+1)-d(i)) .
\end{aligned}
$$

For every $i \in[q-1]$, set $k_{i}=n-d(i)$ and let $k_{q}=n-\left(k_{1}+\ldots+k_{q-1}\right)$. Clearly, $k_{i}>0$ for every $i \in[q]$; also, $k_{1}+\ldots+k_{q}=n$.

Furthermore, for every $h \in[q-2]$, applying (3) with $M_{i}=\Gamma(i), i \in[h]$, and (6), we see that,

$$
\widehat{d}([h])=|\widehat{\Gamma}([h])| \geq \sum_{i=1}^{h} d(i)-(h-1) n=n-\sum_{i=1}^{h} k_{i}>0 .
$$

Hence, by $d(h+1) \leq d(h)$, it follows that

$$
\widehat{d}([h])(d(h+1)-d(h)) \leq\left(n-\sum_{i=1}^{h} k_{i}\right)(d(h+1)-d(h)) .
$$

Since, from (7), we have

$$
d(q) \leq(q-1) n-\sum_{i=1}^{q-1} d(i)=\sum_{i=1}^{q-1} k_{i},
$$

in view of (9) with $h=q-1$, it follows that

$$
\begin{aligned}
\widehat{d}([q-1])(d(q)-d(q-1)) & \leq\left(n-\sum_{i=1}^{q-1} k_{i}\right)(d(q)-d(q-1)) \\
& \leq\left(n-\sum_{i=1}^{q-1} k_{i}\right)\left(\sum_{i=1}^{q-1} k_{i}-d(q-1)\right) .
\end{aligned}
$$


Recalling (8) and (9), this inequality implies that

$$
\begin{aligned}
2 m & \leq n d(1)+\sum_{h=1}^{q-2}\left(n-\sum_{i=1}^{h} k_{i}\right)(d(h+1)-d(h)) \\
& +\left(n-\sum_{i=1}^{q-1} k_{i}\right)\left(\sum_{i=1}^{q-1} k_{i}-d(q-1)\right) .
\end{aligned}
$$

Dividing by 2 and rearranging the right-hand side, we obtain

$$
m \leq\left(n-\sum_{i=1}^{q-1} k_{i}\right)\left(\sum_{i=1}^{q-1} k_{i}\right)+\sum_{1 \leq i<j \leq q-1} k_{i} k_{j}=\sum_{1 \leq i<j \leq q} k_{i} k_{j} .
$$

Note that

$$
\sum_{1 \leq i<j \leq q} k_{i} k_{j}=e\left(K\left(k_{1}, \ldots, k_{q}\right)\right)
$$

Given $n$ and $k_{1}+\ldots+k_{q}=n$, the value $e\left(K\left(k_{1}, \ldots, k_{q}\right)\right)$ attains its maximum if and only if all $k_{i}$ differ by at most 1 , that is to say, when $K\left(k_{1}, \ldots, k_{q}\right)$ is exactly the Turán graph $T_{q}(n)$. Hence, the inequality $m \geq t_{r}(n)$ and (11) imply

$$
t_{r}(n) \leq m \leq e\left(K\left(k_{1}, \ldots, k_{q}\right)\right) \leq t_{q}(n)
$$

Since $q<r \leq n$ implies $t_{q}(n)<t_{r}(n)$, contradicting (12), the proof of $(i)$ is complete.

To prove (ii) suppose (5) fails, i.e.,

$$
\sum_{i=1}^{r} d(i)<(r-1) n
$$

Hence, (10) holds with a strict inequality and so, the proof of $(12)$ gives $t_{r}(n)<t_{r}(n)$. This contradiction completes the proof of (ii).

Proof of (iii) Suppose that for some $\mathcal{P}$-sequence $v_{1}, \ldots, v_{r}, \ldots$ equality holds in (5). We may and shall assume that $v_{1}, \ldots, v_{r}=1, \ldots, r$, i.e.,

$$
\sum_{i=1}^{r} d(i)=(r-1) n
$$

Following the arguments in the proof of (i) and (ii), from (12) we conclude that

$$
t_{r}(n) \leq m \leq t_{r}(n) .
$$

and this completes the proof. 


\section{Degree sums in cliques}

In this section we turn to the problem of finding $\Delta_{r}(n, m)$ for $m \geq t_{r}(n)$. We shall apply Theorem 1 to prove that every graph $G=G(n, m)$ with $m \geq t_{r}(n)$ contains an $r$-clique $R$ with

$$
\sum_{i \in R} d(i) \geq \frac{2 r m}{n} .
$$

As proved by Faudree [7], the required $r$-clique $R$ may be constructed by the algorithm $\mathcal{P}$. Note that the assertion is trivial for regular graphs; as we shall show, if $G$ is not regular, we may demand strict inequality in (13).

Theorem 2 Let $r \geq 2, n \geq r, m \geq t_{r}(n)$ and let $G=G(n, m)$ be a graph which is not regular. Then there exists a $\mathcal{P}$-sequence $v_{1}, \ldots, v_{r}, \ldots$ of at least $r$ terms such that

$$
\sum_{i=1}^{r} d\left(v_{i}\right)>\frac{2 r m}{n} .
$$

Proof Part (iii) of Theorem 1 implies that for some $\mathcal{P}$-sequence, say $1, \ldots, r, \ldots$, we have

$$
\sum_{i=1}^{r} d(i)>(r-1) n
$$

Since $d(i)<n$, we immediately obtain

$$
\sum_{i=1}^{s} d(i)>(s-1) n
$$

for every $s \in[r]$.

The rest of the proof consists of two parts: In part (a) we find an upper bound for $m$ in terms of $\sum_{i=1}^{r} d(i)$ and $\sum_{i=1}^{r} d^{2}(i)$. Then, in part $(b)$, we prove that

$$
\frac{1}{r} \sum_{i=1}^{r} d(i) \geq \frac{2 m}{n},
$$

and show that if equality holds then $G$ is regular.

(a) Partition the set $V$ into $r$ sets $V=V_{1} \cup \ldots \cup V_{r}$, where,

$$
\begin{aligned}
V_{1} & =V \backslash \Gamma(1), \\
V_{i} & =\widehat{\Gamma}([i-1]) \backslash \widehat{\Gamma}([i]) \text { for } i=2, . ., r-1, \\
V_{r} & =\widehat{\Gamma}([r-1]) .
\end{aligned}
$$


We have,

$$
\begin{aligned}
2 m & =\sum_{i \in V} d(i)=\sum_{h=1}^{r} \sum_{j \in V_{h}} d(j) \leq \sum_{i=1}^{r} d(i)\left|V_{i}\right| \\
& =\sum_{i=1}^{r-1}(d(i)-d(r))\left|V_{i}\right|+n d(r)
\end{aligned}
$$

Clearly, for every $i \in[r-1]$, from (3), we have

$$
|\widehat{\Gamma}([i+1])| \geq|\widehat{\Gamma}([i])|+|\Gamma(i+1)|-n=|\widehat{\Gamma}([i])|+d(i+1)-n
$$

and hence, $\left|V_{i}\right| \leq n-d(i)$ holds for every $i \in[r-1]$. Estimating $\left|V_{i}\right|$ in (15) we obtain

$$
\begin{aligned}
2 m & \leq \sum_{i=1}^{r-1}(d(i)-d(r))(n-d(i))+n d(r) \\
& =n \sum_{i=1}^{r} d(i)-\sum_{i=1}^{r} d^{2}(i)+d(r)\left(\sum_{i=1}^{r} d(i)-n(r-1)\right) .
\end{aligned}
$$

(b) Let $S_{r}=\sum_{i=1}^{r} d(i)$. From $d(r) \leq S_{r} / r$ and Cauchy's inequality we deduce

$$
\begin{aligned}
2 m & \leq n S_{r}-\sum_{i=1}^{r} d^{2}(i)+\frac{S_{r}}{r}\left(S_{r}-(r-1) n\right) \\
& \leq n S_{r}-\frac{1}{r}\left(S_{r}\right)^{2}+\frac{S_{r}}{r}\left(S_{r}-(r-1) n\right) \leq \frac{n S_{r}}{r},
\end{aligned}
$$

and so,

$$
\sum_{i=1}^{r} d(i) \geq \frac{2 r m}{n}
$$

To complete the proof suppose we have an equality in (16). This implies that

$$
\sum_{i=1}^{r} d^{2}(i)=\frac{1}{r}\left(\sum_{i=1}^{r} d(i)\right)^{2}
$$

and so, $d(1)=\ldots=d(r)$. Therefore, the maximum degree $d(1)$ equals the average degree $2 m / n$, contradicting the assumption that $G$ is not regular.

Since for every $m \geq t_{r}(n)$ there is a graph $G=G(n, m)$ whose degrees differ by at most 1 , we obtain the following bounds on $\Delta_{r}(n, m)$.

Corollary 1 For every $m \geq t_{r}(n)$

$$
\frac{2 r m}{n} \leq \Delta_{r}(n, m)<\frac{2 r m}{n}+r .
$$




\section{Stability of $\Delta_{r}(n, m)$ as $m$ approaches $t_{r}(n)$}

It is known that inequality (2) is far from being true if $m \leq t_{r}(n)-\varepsilon n$ for some $\varepsilon>0$ (e.g., see [7]). However, it turns out that, as $m$ approaches $t_{r}(n)$, the function $\Delta_{r}(n, m)$ approaches $2 \mathrm{rm} / \mathrm{n}$. More precisely, the following stability result holds.

Theorem 3 For every $\varepsilon>0$ there exist $n_{0}=n_{0}(\varepsilon)$ and $\delta=\delta(\varepsilon)>0$ such that if $m>t_{r}(n)-\delta n^{2}$ then

$$
\Delta_{r}(n, m)>(1-\varepsilon) \frac{2 r m}{n}
$$

for all $n>n_{0}$.

Proof Without loss of generality we may assume that

$$
0<\varepsilon<\frac{2}{r(r+1)}
$$

Set

$$
\delta=\delta(\varepsilon)=\frac{1}{32} \varepsilon^{2} .
$$

If $m \geq t_{r}(n)$, the assertion follows from Theorem 2, hence we may assume that

$$
\frac{2 r m}{n}<\frac{2 r t_{r}(n)}{n} \leq(r-1) n
$$

Clearly, our theorem follows if we show that $m>t_{r}(n)-\delta n^{2}$ implies

$$
\Delta_{r}(n, m)>(1-\varepsilon)(r-1) n
$$

for $n$ sufficiently large.

Suppose the graph $G=G(n, m)$ satisfies $m>t_{r}(n)-\delta n^{2}$. By (4), if $n$ is large enough,

$$
m>t_{r}(n)-\delta n^{2}>\left(\frac{r-1}{2 r}-\delta\right) n^{2}-\frac{r}{8} \geq\left(\frac{r-1}{2 r}-2 \delta\right) n^{2} .
$$

Let $M_{\varepsilon} \subset V$ be defined as

$$
M_{\varepsilon}=\left\{u: d(u) \leq\left(\frac{r-1}{r}-\frac{\varepsilon}{2}\right) n\right\} .
$$

The rest of the proof consists of two parts. In part (a) we shall show that $\left|M_{\varepsilon}\right|<\varepsilon n$, and in part (b) we shall show that the subgraph induced by $V \backslash M_{\varepsilon}$ contains an $r$-clique with large degree sum, proving (17).

(a) Our first goal is to show that $\left|M_{\varepsilon}\right|<\varepsilon n$. Indeed, assume the opposite and select an arbitrary $M^{\prime} \subset M_{\varepsilon}$ satisfying

$$
\left(\frac{1}{2}-\frac{1}{2 \sqrt{2}}\right) \varepsilon n<\left|M^{\prime}\right|<\left(\frac{1}{2}+\frac{1}{2 \sqrt{2}}\right) \varepsilon n .
$$


Let $G^{\prime}$ be the subgraph of $G$ induced by $V \backslash M^{\prime}$. Then

$$
\begin{aligned}
e(G) & =e\left(G^{\prime}\right)+e\left(M^{\prime}, V \backslash M^{\prime}\right)+e\left(M^{\prime}\right) \leq e\left(G^{\prime}\right)+\sum_{u \in M^{\prime}} d(u) \\
& \leq e\left(G^{\prime}\right)+\left|M^{\prime}\right|\left(\frac{r-1}{r}-\frac{\varepsilon}{2}\right) n .
\end{aligned}
$$

Observe that second inequality of (19) implies

$$
n-\left|M^{\prime}\right|>(1-\varepsilon) n .
$$

Hence, if

$$
e\left(G^{\prime}\right) \geq \frac{r-1}{2 r}\left(n-\left|M^{\prime}\right|\right)^{2}
$$

then, applying Theorem 2 to the graph $G^{\prime}$, we see that

$$
\Delta_{r}(G) \geq \Delta_{r}\left(G^{\prime}\right) \geq \frac{2 r e\left(G^{\prime}\right)}{n-\left|M^{\prime}\right|} \geq(r-1)\left(n-\left|M^{\prime}\right|\right)>(r-1)(1-\varepsilon) n,
$$

and (17) follows. Therefore, we may assume

$$
e\left(G^{\prime}\right)<\frac{r-1}{2 r}\left(n-\left|M^{\prime}\right|\right)^{2} .
$$

Then, by (18) and (20),

$$
\frac{r-1}{2 r}\left(n-\left|M^{\prime}\right|\right)^{2}>e\left(G^{\prime}\right)>-\left|M^{\prime}\right|\left(\frac{r-1}{r}-\frac{\varepsilon}{2}\right) n+\left(\frac{r-1}{2 r}-2 \delta\right) n^{2} .
$$

Setting $x=\left|M^{\prime}\right| / n$, this shows that

$$
\frac{r-1}{2 r}(1-x)^{2}+x\left(\frac{r-1}{r}-\frac{\varepsilon}{2}\right)-\left(\frac{r-1}{2 r}-2 \delta\right)>0,
$$

which implies that

$$
x^{2}-\varepsilon x+4 \delta>0 .
$$

Hence, either

$$
\left|M^{\prime}\right|>\left(\frac{\varepsilon-\sqrt{\varepsilon^{2}-16 \delta}}{2}\right) n=\left(\frac{1}{2}-\frac{1}{2 \sqrt{2}}\right) \varepsilon n
$$

or

$$
\left|M^{\prime}\right|<\left(\frac{\varepsilon+\sqrt{\varepsilon^{2}-16 \delta}}{2}\right)=\left(\frac{1}{2}+\frac{1}{2 \sqrt{2}}\right) \varepsilon n,
$$

contradicting (19). Therefore, $\left|M_{\varepsilon}\right|<\varepsilon n$, as claimed

(b) Let $G_{0}$ be the subgraph of $G$ induced by $V \backslash M_{\varepsilon}$. By the definition of $M_{\varepsilon}$, if $u \in$ $V \backslash M_{\varepsilon}$, then

$$
d_{G}(u)>\left(\frac{r-1}{r}-\frac{\varepsilon}{2}\right) n,
$$


and so

$$
d_{G_{0}}(u)>\left(\frac{r-1}{r}-\frac{\varepsilon}{2}\right) n-\left|M_{\varepsilon}\right|>\frac{r-2}{r-1}\left(n-\left|M_{\varepsilon}\right|\right) .
$$

Hence, by Turán's theorem, $G_{0}$ contains an $r$-clique and, therefore,

$$
\Delta_{r}(G)>r\left(\frac{r-1}{r}-\frac{\varepsilon}{2}\right) n \geq(1-\varepsilon)(r-1) n,
$$

proving (17) and completing the proof of our theorem.

Acknowledgement. The authors are grateful to Prof. D. Todorov for pointing out a fallacy in an earlier version of the proof of Theorem 2 and to the referee for his valuable suggestions.

Added on July 1st, 2005. The results of this paper were first presented in a seminar at Memphis University in February, 2002 and also form part of the second author's Ph.D. thesis [10], Ch. 7. The results in Theorems 1 and 2 were reproduced by Khadzhiivanov and Nenov in [8], [9].

\section{References}

[1] B. Bollobás, Modern Graph Theory, Graduate Texts in Mathematics 184, Springer Verlag, 1998, xiv-394pp.

[2] B. Bollobás and P. Erdős, Unsolved problems, Proc. Fifth Brit. Comb. Conf. (Univ. Aberdeen, Aberdeen, 1975), Winnipeg, Util. Math. Publ., 678-680.

[3] C. Edwards, The largest vertex degree sum for a triangle in a graph, Bull. Lond. Math. Soc., 9 (1977), 203-208.

[4] C. Edwards, Complete subgraphs with largest sum of vertex degrees, Combinatorics (Proc. Fifth Hungarian Colloq., Keszthely, 1976), Vol. I, Colloq. Math. Soc. János Bolyai, 18, North-Holland, Amsterdam-New York, 1978, pp. 293-306.

[5] P. Erdős and R. Laskar, On maximum chordal subgraph, Proceedings of the fourteenth Southeastern conference on combinatorics, graph theory and computing (Boca Raton, Fla., 1983). Congr. Numer. 39 (1983), 367-373.

[6] G. Fan, Degree sum for a triangle in a graph, J. Graph Theory 12 (1988), 249-263.

[7] R. Faudree, Complete subgraphs with large degree sums, J. Graph Theory 16 (1992), 327-334.

[8] N. Khadzhiivanov and N. Nenov, Sequences of maximal degree vertices in graphs, Serdica Math. J. 30 (2004), 95-102.

[9] N. Khadzhiivanov and N. Nenov, Saturated $\beta$-sequences in graphs, C. R. Acad. Bulgare Sci. 57 (2004), 49-54.

[10] V. Nikiforov, Stability results in extremal graph theory, PhD thesis, Inst. of Math. and Inform., Bul. Acad. Sci., Sofia. 